Communication

\title{
Studies on Photodecomposition of an Oxime Sulfonate
}

\author{
Toshikage Asakura ${ }^{a, c}$, Hitoshi Yamato ${ }^{\text {b }}$, Keita Tanaka ${ }^{b}$, Ryuichi Takahashi ${ }^{\text {b }}$ \\ Hisatoshi Kura ${ }^{a}$ and Tamaki Nakano ${ }^{c}$
}

\author{
${ }^{a}$ Development Electronic Specialties, Dispersions and Pigments AsiaPacific, BASF Japan Ltd. \\ ${ }^{b}$ Organic Electronics Research, BASF Japan Ltd. 7-1-13, Doicho, Amagasaki-shi, Hyogo,660-0083,Japan \\ ${ }^{c}$ Catalysis Research Center (CRC) and Graduate School of Chemical Sciences and Engineering \\ Hokkaido University, N21, W10, Kita-ku, Sapporo, 001-0021, Japan
}

Keywords: photodecomposition, oxime sulfonate, g-line, i-line, photoacid generator

\section{Introduction}

Since chemical amplified (CA) photoresist system has been widely accepted by semiconductor manufacturers to accomplish their high resolution requirement, photoacid generators (PAGs) have been playing one of the most important roles among photoresist components. These days, CA resist system has been employed not only for semiconductor device fabrication but also for micro electrical mechanical system (MEMS) preparation. [1]

Though we have been studying and reporting oxime sulfonates as PAG mainly regarding properties related to photolithography, its photoreaction has not been discussed enough so far. [2]

In this paper, photodecomposition of a photoacid generator, (2-propylsulfonyloxyimino- $2 H$-thiophen3-ylidene)-2-methylphenyl-acetonitrile (2-PTMA), is studied and reported from viewpoints of change of UV absorption determined by UV spectrometer, components and mass determination of the photochemically decomposed material by Gas Chromatography and NMR spectroscopic analyses of photochemically generated compounds before and after separation by column chromatography. Finally Compounds generated by photodecomposition are identified.

\section{Experimental}

2.1 Change of UV absorption under exposure

All the chemicals, solvents, materials in this paper were used as obtained without further purification processes. $50 \mathrm{mg}$ of 2-PTMA (commercially available as Irgacure PAG 103 from

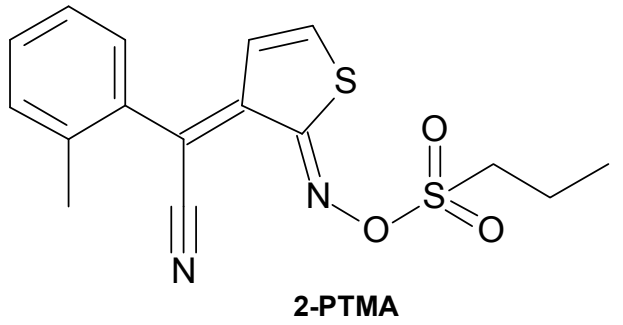

Scheme 1

BASF Japan Ltd.) was dissolved in $5 \mathrm{~mL}$ of acetonitrile (LC/MS grade from Wako, Japan) to form $10 \mathrm{mg} / \mathrm{mL}$ solution. Ca. $4 \mathrm{~mL}$ of the solution was exposed in an optical cuvette by UV radiation from a high pressure mercury lamp (PM25C-110/ HB-25103BY-C from Ushio, Japan) for two hours. $50 \mu \mathrm{L}$ of the solution was sampled periodically to observe the change of UV absorption in an optical cuvette with $1 \mathrm{~cm}$ optical path length by UV/ visible spectrometer (U-3310 from Hitachi, Japan) after dilution to $0.01 \mathrm{mg} / \mathrm{mL}$ concentration.

\subsection{Gas Chromatography}

The 2-PTMA solutions before and after exposure processes were analyzed with gas chromatography (7890B form Agilent Technologies, US) detected by EI-low resolution mass spectrometer (5977A from Agilent Technologies, US) Two detection methods, TIC and FID, were employed.

$\mathrm{GC} / \mathrm{mass}$ conditions are listed below;

$\begin{array}{ll}\text { Column } & \text { RTX-200MS (from Restek, US) } \\ \text { Temperature } & 50-300{ }^{\circ} \mathrm{C} \\ \text { Heating rate } & 10{ }^{\circ} \mathrm{C} / \mathrm{min} \\ \mathrm{m} / \mathrm{z} & 35-600\end{array}$


2.3 Separation of the decomposed compounds

The decomposed compounds photochemically produced were separated by flash column chromatography after preliminary analysis with thin layer chromatography (TLC). The decomposed compounds were separated by a normal phase column chromatogram filled with silica gel. The eluent employed here graduated from 1:20 of ethyl acetate: hexane to 1:5 and fractioned by $20 \mathrm{~mL}$ each. Each of the collected fractions was confirmed that it included only a single compound by thin layer chromatography and consolidated appropriately.

\section{$2.4{ }^{1} \mathrm{H}$ and ${ }^{13} \mathrm{C}$ NMR spectroscopies}

The mixture and the separated photochemically decomposed compounds were analyzed by ${ }^{1} \mathrm{H}$ and ${ }^{13} \mathrm{C}$ NMR spectroscopy (Varian NMR system $400 \mathrm{MHz}$ from Varian, US) in deuterated chloroform.

In addition to 1D spectroscopy, 2D spectroscopic measurements were conducted by ${ }^{1} \mathrm{H}-{ }^{1} \mathrm{H}$ correlation spectroscopy (COSY), ${ }^{1} \mathrm{H}-{ }^{1} \mathrm{H}$ NOE correlated spectroscopy (NOESY), ${ }^{1} \mathrm{H}-{ }^{13} \mathrm{C}$ hetero-nuclear single quantum correlation spectroscopy (HSQC) and ${ }^{1} \mathrm{H}^{13} \mathrm{C}$ hetero-nuclear multiple-bond correlation spectroscopy (HMBC) to determine the chemical structures of the compounds.

\section{Results and Discussion}

3.1 Change of UV absorption determined by UV spectrometer

The absorption spectra were plotted in Figure 1. The main absorption at long wavelength with the $\lambda_{\text {max }}$ at $405 \mathrm{~nm}$ decreased with exposure and a new peak at $357 \mathrm{~nm}$ and distinctive absorption at $265 \mathrm{~nm}$ were observed. No major change in UV absorption was observed after 30 minutes exposure under the experimental conditions in this report. The tendency found here was basically the same as the ones with (2-methylsulfonyloxyimino$2 H$-thiophen-3-ylidene)-2-methylphenyl-acetonitrile (MTMA) reported previously. [2] The fact that clear isocratic points are observed indicates the photochemical decomposition reaction is one specific reaction and no further reaction takes place.

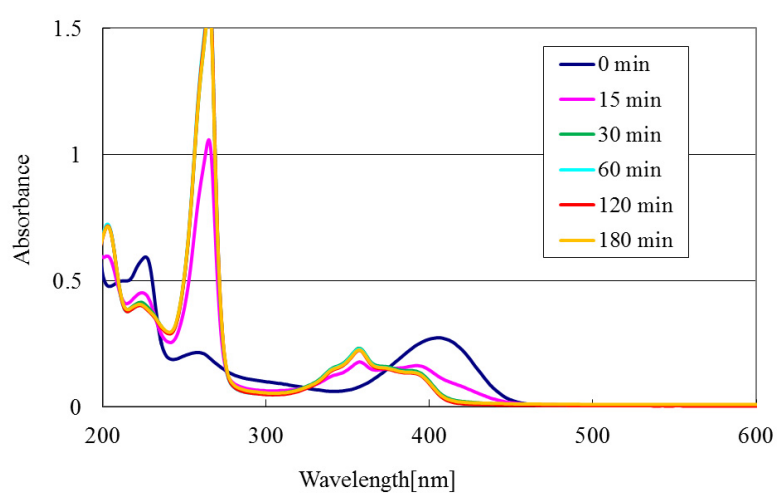

Fig. 1. UV absorption change of 2-PTMA under UV exposure

\subsection{Gas Chromatography}

The experimental results of gas chromatography detected by mass were plotted in Figure 2 . Interestingly it was found that the decomposition of 2-PTMA photochemically generates a 1:1 mixture of two compounds. Moreover the molecular weight of the two compounds was same, $224 \mathrm{~g} / \mathrm{mol}$.
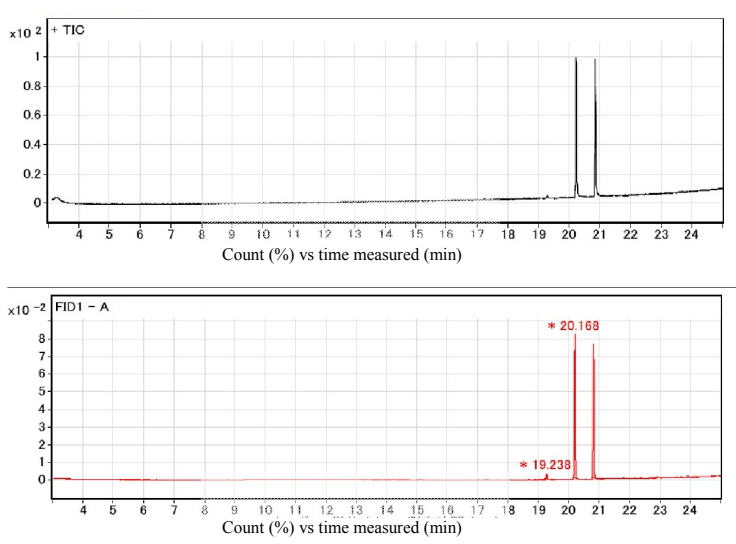

Fig. 2. Analysis results with GC-mass detecting TIC (upper) and FID (lower)

\subsection{Separation of the decomposed compounds}

In TLC analysis prior to column separation, the two components were clearly separated. Tentatively the compound eluted first is named as "Product A" and the other as "Product B". Rf value of Product A and B was 0.44 and 0.31 with ethyl acetate and hexane mixture at 1:9 ratio by volume as a eluent, respectively.

They were separated and purified by flash column chromatography and analyzed by UV/visible spectroscopy, GC mass and ${ }^{1} \mathrm{H}$ and ${ }^{13} \mathrm{C}$ NMR spectroscopy in detail. They exhibited similar UV 
absorption profiles and their molecular weight was confirmed as $224 \mathrm{~g} / \mathrm{mol}$. UV-absorption properties are listed in Table 1. The facts obtained here clearly suggest that the two compounds are structural isomers each other exhibiting extended conjugation.

Table 1. UV absorption peaks of the compounds

\begin{tabular}{ll}
\hline Product & $\lambda_{\max }\left(\varepsilon_{\max }\right)$ \\
\hline $\mathrm{A}$ & $256 \mathrm{~nm}(60000), 357 \mathrm{~nm}(8400)$ \\
$\mathrm{B}$ & $256 \mathrm{~nm}(57000), 357 \mathrm{~nm}(8100)$ \\
\hline
\end{tabular}

\section{$3.4{ }^{1} \mathrm{H}$ and ${ }^{13} \mathrm{C}$ NMR spectroscopy}

Product A and B were analyzed by various NMR spectroscopies. COSY informed coupling among protons and NOESY indicated steric information of protons close each other. Direct bonding correlations of a carbon and a proton were informed by HSQC and sequences information of carbon-carbon bonding was determined by the experimental results with $\mathrm{HMBC}$.

Figure 3 shows the HMBC spectrum of Product $A$ as an example. The correlations of the observed chemical shifts for both products of A and B with their assignments are summarized and listed in Table 2 for the ${ }^{1} \mathrm{H}$ and in Table 3 for ${ }^{13} \mathrm{C}$ NMR spectra.

In ${ }^{1} \mathrm{H}$ NMR studies, it is obvious that all the protons except ones of the methyl group are located in aromatic region which shows good match with the UV absorption indicating a long conjugation. Experimental results from COSY and NOESY indicates that the tolyl ring of the PAG is remaining after reaction but fused at two carbon, the next carbon to methyl position and the one next to that, in both Product A and B and aromaticity is preserved. Each of the hydrogen atoms on the tolyl rings is identified. The authors concluded in this paper that the proton of the ethenylene with the chemical shift at higher magnetic field is located next to sulfur referring the experimental results reported by Klemm et al. with 4-nitro-thienopyridine derivatives. [3] However it is notable that assignments of $\mathrm{H}-2$ and $\mathrm{H}-3$ may be reversed.

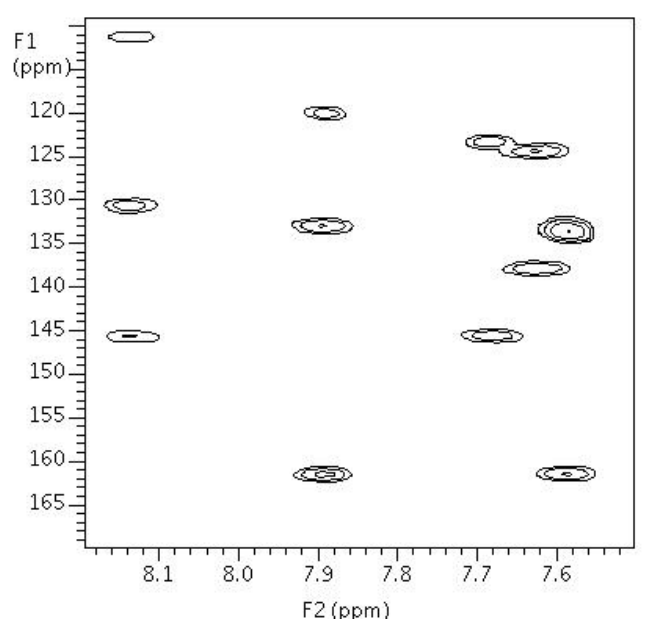

Fig. 3 HMBC spectrum of Product A

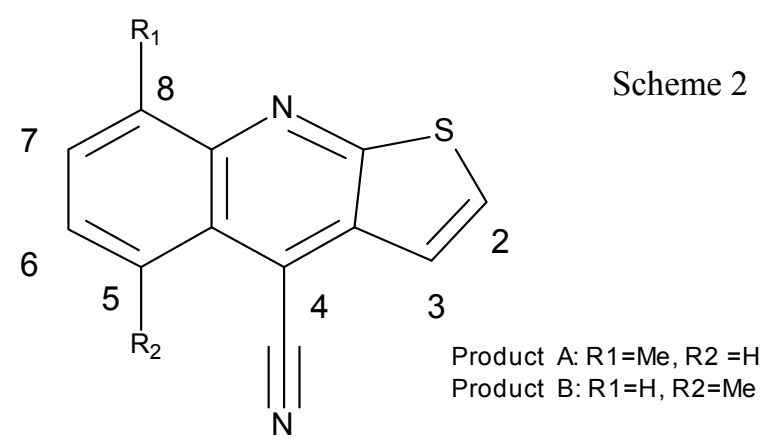

In cases of ${ }^{13} \mathrm{C}$ NMR, the results from HSQC indicate the carbons directly attaching to hydrogen atoms and those from HMBS indicate long range coupling in 2-3 carbon sequences. Especially, even

Table 2. ${ }^{1} \mathrm{H}$ NMR spectral data of Product A and B

\begin{tabular}{|c|c|c|c|c|c|c|c|c|c|c|}
\hline \multirow[t]{2}{*}{.${ }^{1} \mathrm{H}$ atom } & $\begin{array}{l}\text { Product } \\
\text { A }\end{array}$ & \multicolumn{9}{|c|}{ Product B } \\
\hline & $\begin{array}{l}\text { chemical } \\
\text { shift } \\
(\mathrm{ppm})\end{array}$ & signal & $\begin{array}{l}\text { coupler(s) } \\
\text { (COSY) }\end{array}$ & $\begin{array}{l}\text { coupling } \\
(\mathrm{Hz})\end{array}$ & $\begin{array}{l}\text { Coupler } \\
\text { (NOESY) }\end{array}$ & $\begin{array}{l}\text { chemical } \\
\text { shift } \\
(\mathrm{ppm})\end{array}$ & signal & $\begin{array}{l}\text { coupler(s) } \\
\text { (COSY) }\end{array}$ & $\begin{array}{c}\text { coupling } \\
(\mathrm{Hz})\end{array}$ & $\begin{array}{l}\text { coupler } \\
\text { (NOESY) }\end{array}$ \\
\hline $\mathrm{CH} 3$ & 2.90 & $S$ & & & $\mathrm{H}-7$ & 3.14 & $\mathrm{~s}$ & & & H-6 \\
\hline$H-2^{\text {a) }}$ & 7.60 & $\mathrm{D}$ & $\mathrm{H}-3$ & 6.0 & & 7.64 & $\mathrm{~d}$ & $\mathrm{H}-3$ & 6.0 & \\
\hline $\mathrm{H}-3^{\text {a) }}$ & 7.90 & $\mathrm{D}$ & $\mathrm{H}-2$ & 6.4 & & 7.88 & $\mathrm{~d}$ & $\mathrm{H}-2$ & 6.4 & \\
\hline H-5 & 8.16 & $\mathrm{D}$ & H-6 & 8.4 & & & & & & \\
\hline H-6 & 7.64 & $\mathrm{~T}$ & H-5,H-7 & 7.6 & & 7.48 & $\mathrm{~d}$ & $\mathrm{H}-7$ & 6.8 & $\mathrm{CH} 3$ \\
\hline $\mathrm{H}-7$ & 7.69 & $\mathrm{D}$ & H-6 & 6.8 & $\mathrm{CH} 3$ & 7.69 & $\mathrm{t}$ & H-6, H-8 & 7.8 & \\
\hline H-8 & & & & & & 8.07 & $\mathrm{~d}$ & $\mathrm{H}-7$ & 8.4 & \\
\hline
\end{tabular}

a) Assignments of H-2 and H-3 may be reversed. 
Table $3 .{ }^{13} \mathrm{C}$ NMR spectral data of Product A and B

\begin{tabular}{|c|c|c|c|c|}
\hline $\begin{array}{l}{ }^{13} \mathrm{C} \\
\text { atom }\end{array}$ & $\begin{array}{l}\text { Product } \\
\text { A }\end{array}$ & & $\begin{array}{l}\text { Product } \\
\text { B }\end{array}$ & \\
\hline & $\begin{array}{l}\text { chemical } \\
\text { shift } \\
(\mathrm{ppm})\end{array}$ & coupler(s) & $\begin{array}{l}\text { chemical } \\
\text { shift } \\
(\mathrm{ppm})\end{array}$ & coupler(s) \\
\hline $\mathrm{CH} 3$ & 18.28 & H-7 & 22.32 & H-6 \\
\hline $\mathrm{CN}$ & 115.38 & & 117.94 & \\
\hline$C-2^{\text {a) }}$ & 119.71 & $\mathrm{H}-3$ & 120.70 & $\mathrm{H}-3$, \\
\hline $\mathrm{C}-3^{\text {a) }}$ & 133.59 & H-2, & 133.46 & $\mathrm{H}-2$, \\
\hline$C-3 a$ & 132.64 & H-2, H-3, & 135.10 & H-2, H-3, \\
\hline C-4 & 110.89 & $\mathrm{H}-5$, & 109.26 & \\
\hline C-4a & 124.03 & H-6, & 122.78 & $\begin{array}{l}\text { H-6, H-8, } \\
\text { CH3 }\end{array}$ \\
\hline C-5 & 123.00 & $\mathrm{H}-7$, & 134.36 & $\mathrm{H}-7, \mathrm{CH} 3$ \\
\hline C-6 & 128.07 & & 130.25 & $\begin{array}{l}\mathrm{H}-7, \mathrm{H}-8, \\
\mathrm{CH} 3\end{array}$ \\
\hline C-7 & 130.26 & $\mathrm{H}-5, \mathrm{CH} 3$ & 129.77 & \\
\hline C-8 & 137.59 & $\mathrm{H}-6, \mathrm{CH} 3$ & 128.38 & H-6, \\
\hline C-8a & 145.34 & $\begin{array}{l}\text { H-5, H-7, } \\
\text { CH3 }\end{array}$ & 147.22 & H-7, \\
\hline C-9a & 161.22 & H-2, H-3, & 161.19 & $\mathrm{H}-2, \mathrm{H}-3$ \\
\hline
\end{tabular}

in 1D spectroscopy, the carbons locating at distinctively low magnetic field, around $161 \mathrm{ppm}$ for C-9a and 145-147 ppm for C-8a, showed good match with the results previously reported [4] and are key to determine the baseline structure of the products.

Authors finally conclude that a 4-cyanothienoquinoline base-structure reasonably explains the experimental results obtained without any contradiction and that the chemical structure of Product A and B are shown in Scheme 2.

\section{References}

1. H. Ito, C. G. Wilson, Proseedings of the SPE Regional Technical Conference on Photopolymers, Society of Plastics Engineers, Nov. (1982) 331.

2. T. Asakura, H. Yamato, M. Ohwa, J. Photopolym. Sci. Technol., 13 (2000) 223.

3. L. H. Klemm, J. Wang, S. K. Sur, J. Heterocyclic Chem., 27 (1990) 1537.

4. L. H. Klemm, D. R. Muchiri, J. N. Louris, J. Heterocyclic Chem., 21 (1985) 1135. 